\title{
Characterisation of 3-beta-hydroxysteroid dehydrogenase from pig liver and testis in relation
}

to boar taint

S. I. Nicolau-Solano, F. M. Whittington, J. D. Wood, and E. Doran

Department of Clinical Veterinary Science, School of Veterinary Sciences, University of Bristol, Langford, Bristol, BS40 5DU, U.K. Email: silvia.nicolau@bristol.ac.uk

Introduction Boar taint is a major meat quality defect, which affects about $10 \%$ of entire male pigs. It is due to an excessive accumulation of skatole and androstenone in adipose tissue. One of the reasons for accumulation of these compounds is a low rate of their metabolism. Androstenone is metabolised in liver via the enzyme 3-betahydroxysteroid dehydrogenase (HSD). This enzyme is well characterised in the testis, where it participates in the synthesis of steroids, while its properties in liver are unknown. The aim of the present study was to characterise and compare properties of HSD from pig liver versus pig testis when metabolising androstenone.

Materials and methods Liver and testis samples were collected from 5 Large-White pigs immediately after slaughter. Microsomes were isolated by differential centrifugation and frozen at $-80^{\circ} \mathrm{C}$ until use. Microsomes were incubated under different conditions in order to determine HSD kinetic parameters and metabolic rates with androstenone as substrate and NADH as cofactor. Rates were estimated by recording the appearance of the main metabolite, $\beta$ androstenol ( $\beta$-ol), by high-resolution gas chromatography. For kinetic experiments the incubation conditions were: 37 ${ }^{\circ} \mathrm{C}, \mathrm{pH} 7.4$ and incubation time $20 \mathrm{~min}$, except when different $\mathrm{pH}$ of the incubation media were used for determining the optimum $\mathrm{pH}$. Different concentrations of androstenone as substrate and trilostane as a HSD specific inhibitor were used. The amount of HSD protein in isolated microsomes was determined by Western Blotting using specific antibodies. ANOVA was used for the statistical analysis.

Results The rate of androstenone metabolism was higher in liver than in testis (Figure 1). The kinetic study revealed that hepatic HSD has lower affinity for androstenone as a substrate in comparison with testicular HSD. However, hepatic HSD is able to metabolise androstenone faster than the testicular enzyme. Differences were observed in the optimum $\mathrm{pH}$ range as well (Table 1). Inhibition studies showed that hepatic HSD was more sensitive to trilostane than testicular HSD (Figure2). In both cases competitive inhibition occurred. Western Blotting results showed no differences in the amount of HSD protein in pig liver and testis (Table1).



Fig 1 HSD time-dependent curve for androstenone metabolism

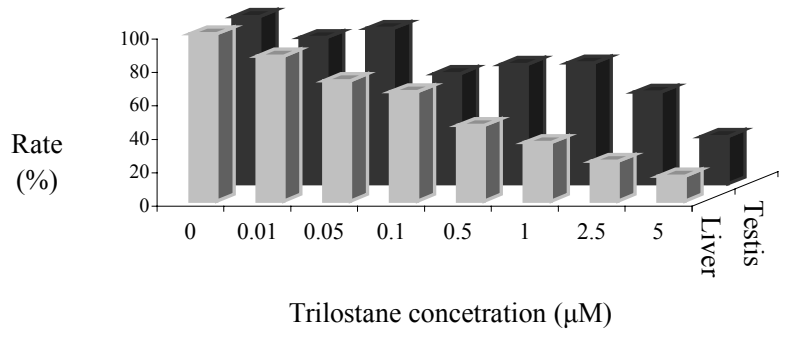

Fig 2 Trilostane effect on hepatic and testicular HSD

Table 1 Metabolic rates, kinetic parameters and expression of HSD in pig liver and testis

\begin{tabular}{lll}
\hline Parameter & Liver & \multicolumn{1}{c}{ Testis } \\
\hline Metabolic rate $(\mu \mathrm{g} \beta-\mathrm{ol} / \mathrm{mg}$ prot/min) & $1.28^{*}$ & $0.24^{*}$ \\
$\mathrm{Km}(\mu \mathrm{M})$ & $253^{*}( \pm 38.9)$ & $59^{*}( \pm 24.3)$ \\
Vmax $(\mu \mathrm{g} \beta-\mathrm{ol} / \mathrm{mg} \mathrm{prot} / \mathrm{min})$ & $2.3^{*}( \pm 0.5)$ & $0.5^{*}( \pm 0.1)$ \\
Optimum pH & 7 & 6 \\
Kind of inhibition of trilostane & competitive & competitive \\
Amount of HSD (arbitrary units) & $90( \pm 4)$ & $92( \pm 9)$ \\
\hline
\end{tabular}

(* denotes statistical significance, $\mathrm{p}<0.05)$

Conclusions The results of this study demonstrate that HSD activity and kinetic characteristics differ in pig liver and testis. However, the amount of HSD protein expression was similar in both tissues. Therefore we conclude that there might be different HSD isoforms in pigs and their distribution is tissues specific. This study will be extended to different breeds in order to identify an apparent polymorphism in HSD. Such a polymorphism could be used to develop a genetic test for boar taint.

\section{Acknowledgements}

The author wishes to thanks to BBRSC, Genesis Faraday and Meat Livestock Commission for their financial contribution to this work as well as Stegram Pharmaceuticals for the kind donation of trilostane. 